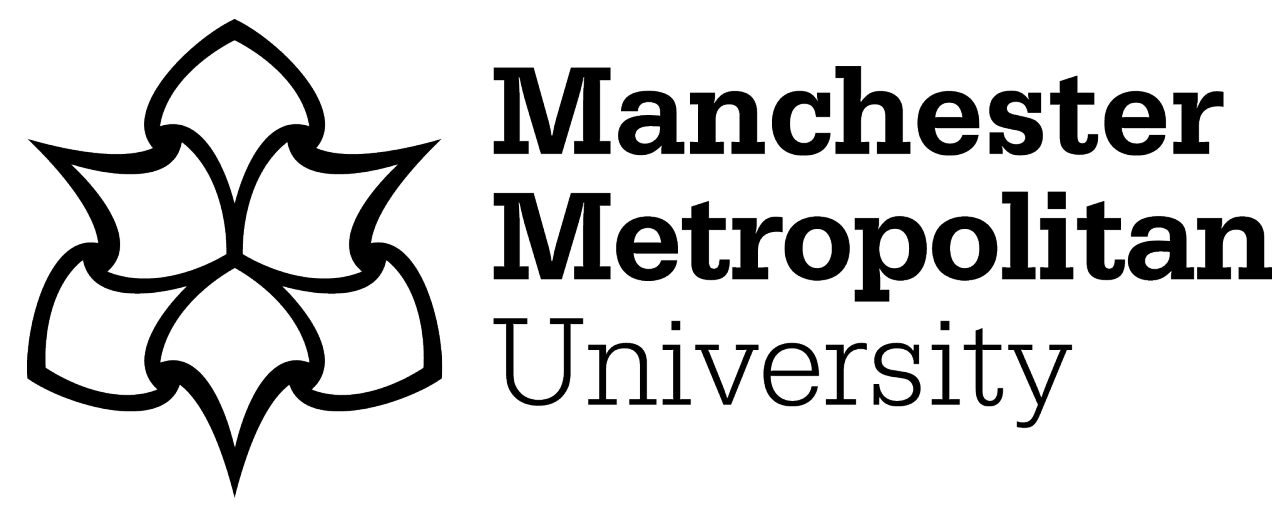

Riaz, F, Naeem, S, Nawaz, Raheel and Coimbra, MT (2018) Active Contours Based Segmentation and Lesion Periphery Analysis For Characterization of Skin Lesions in Dermoscopy Images. IEEE Journal of Biomedical and Health Informatics, 23 (2). pp. 489-500. ISSN 2168-2194

Downloaded from: https://e-space.mmu.ac.uk/620994/

Version: Accepted Version

Publisher: IEEE

DOI: https://doi.org/10.1109/JBHI.2018.2832455

Please cite the published version 


\title{
Active Contours Based Segmentation and Lesion Periphery Analysis For Characterization of Skin Lesions in Dermoscopy Images
}

\author{
Farhan Riaz, Sidra Naeem, Raheel Nawaz and Miguel Coimbra
}

\begin{abstract}
This paper proposes a computer assisted diagnostic (CAD) system for the detection of melanoma in dermoscopy images. Clinical findings have concluded that in case of melanoma, the lesion borders exhibit differential structures such as pigment networks and streaks as opposed to normal skin spots, which have smoother borders. We aim to validate these findings by performing segmentation of the skin lesions followed by an extraction of the peripheral region of the lesion that is subjected to feature extraction and classification for detecting melanoma. For segmentation, we propose a novel active contours based method that takes an initial lesion contour followed by the usage of Kullback-Leibler divergence between the lesion and skin to fit a curve to the lesion boundaries. After segmentation of the lesion, its periphery is extracted to detect melanoma using image features that are based on local binary patterns. For validation of our algorithms, we have used the publicly available $\mathbf{P H}^{2}$ and ISIC dermoscopy datasets. An extensive experimental analysis reveals two important findings: 1 . The proposed segmentation method mimics the ground truth data, and 2 . The most significant melanoma characteristics in the lesion actually lie on the lesion periphery.
\end{abstract}

Index Terms-Image Segmentation, Active Contours, Local Binary Patterns, Classification, Dermoscopy.

\section{INTRODUCTION}

$\mathbf{M}$ ELANOMA is the most deadly form of skin cancer that comprises about $4.5 \%$ of all cancers that affect humans [1]. The incidence of melanoma results in a survival rate of only about $15 \%$ in the first five years [2]. Given that the incidence rate of melanoma has been increasing over the past three decades [3] and its moratlity rate is very high if not diagnosed at an early stage, early detection of melanoma is of significant importance. Assisted diagnostic tools can help significantly in early detection of cancer and thus, an effective recovery of the patients. Dermoscopy is a non-invasive technique that is used for the detection of melanoma. It involves visual inspection of the pigmented skin lesion by placing gel on the affected skin, followed by its observation with the help of a magnification instrument called a dermoscope that has

F. Riaz is with Department of Computer Engineering, National University of Sciences and Technology, Islamabad, Pakistan (e-mail: farhan.riaz@ce.ceme.edu.pk).

S. Naeem is with Department of Electrical Engineering, COMSATS Institute of Information Tehcnology, Wah, Pakistan (e-mail: sidranaeem@ciitwah.edu.pk).

R. Nawaz is Reader in Text and Data Mining, Manchester Metropolitan University, Manchester, UK (e-mail: r.nawaz@mmu.ac.uk).

M. Coimbra is with the Instituto de Telecomunicações, Department of Computer Science, Faculdade de Ciências da Universidade do Porto, 4169-007 Porto, Portugal (e-mail: mcoimbra@dcc.fc.up.pt). the ability to amplify a skin image by upto 100 times. The dermoscope allows a detailed inspection of the subsurface structures of the skin followed by the application of diagnostic methods for a clinical assessment of the lesion.

There are four main diagnostic methods to detect melanoma: the ABCD rule, pattern analysis, Menzie's method and the seven-point check list. These methods have been validated at the 2000 Consensus Net Meeting on Dermoscopy (CNMD) [4] by experts from across the world and are widely used by the physicians for diagnostic purposes. All these methods are based on the visual characteristics of the skin lesions. It has been validated that dermoscopy can increase the sensitivity of melanoma detection by about 10\%-27\% [5]. Researchers have found that dermoscopy increases diagnostic performance only if formal training has been received by dermatologists [5]. If the dermatologist is not adequately trained, there is an inverse relation between the use of dermoscopy and its diagnostic effectiveness towards early detection of melanoma [6]. Furthermore, the human analysis is subjective since it depends on human vision and clinical experience [7] making the diagnosis of melanoma difficult to reproduce. This has led to the emergence of CAD systems that can facilitate in diagnosing melanomas by handling all these issues and providing a second opinion which can be potentially very useful for its early detection.

Rest of the paper is organized as follows: We discuss the related work and highlight our contributions in Section II, followed by discussion of the proposed segmentation method in Section III. Later, we discuss feature extraction and classification methodology in Section IV, perform our experiments with a visual analysis of the segmentation results (Section V) and conclude the paper (Section VI).

\section{BACKGROUND}

A melanocytic lesion can be identified by its general dermoscopic pattern that can be referred to as a global pattern, whereas a more detailed description of the lesion can be performed by the analysis of its local patterns. The global features are basically composed of a repetitive arrangement of micro-texture patterns such as streaks, pigment networks, blue-white veils, dots and globules [8]. Several visual features can be used for the detection of melanoma [9], texture being the most important one. The main objective of this paper is to classify a dermoscopic lesion as being either normal or cancerous (melanoma). A pattern recognition (PR) system that 
has the ability to classify the skin lesions is usually composed of three stages: 1). segmentation, 2). feature extraction, and 3 ). classification. Given that the main focus of the paper is on image segmentation followed by a study of the impact of lesion periphery on diagnostics in dermoscopy, we visit the relevant literature on segmentation of dermoscopy images and clinical background on the significance of lesion periphery.

\section{A. Background on dermoscopic image segmentation}

Segmentation is a vital step in automated diagnostic systems for melanoma as it affects all the subsequent stages for the design of effective CAD systems. It is also a very challenging problem due to various factors including illumination variations, irregular structural and color variations, presence of hair and existence of multiple lesions in a close vicinity. Several methods have been proposed to tackle this important task. In the context of dermoscopic image analysis, the segmentation methods can be mainly categorized as: thresholding, region based and edge based methods [10]-[12].

Indeed, the simplest skin lesion segmentation is thresholding [13]-[17]. The thresholding techniques work well in cases where there is a good contrast between the lesion and the skin, ideally yielding bimodal image histograms. When the histogram modes corresponding to the skin and the lesion overlap, segmentation becomes a challenge and cannot be handled using thresholding. Another category of algorithms are the region based methods. Some examples of these methods include multiscale region growing [14] and region merging [18]. The region based methods have difficulties in effective segmentation when the lesion is textured or inhomogeneous [19]. This is very common since the periphery of the lesion contains dermoscopic differential structures that can result in an oversegmentation of the image (Fig. 1-left). Sometimes, a soft skin tone change happens as we traverse from the lesion to the skin leading to imprecise segmentation (Fig. 1-right).

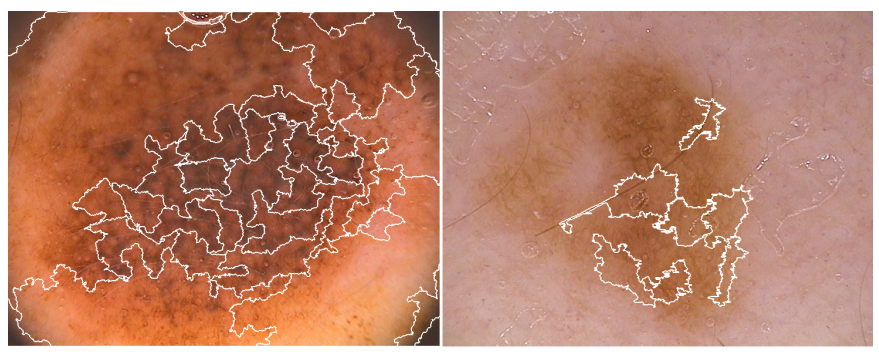

Fig. 1: A visual illustration of over segmentation of a highly textured lesion (left) and under segmentation of a lesion having low contrast from the skin.

Last but not the least, another important class of segmentation methods is the edge based methods. Examples of such methods include the watersheds [20]-[22], morphological flooding [12], [23] etc. These methods rely heavily on the edge features of the images that segregate the object from the background. In the context of the segmentation of dermoscopy images, these methods are expected to perform poorly when the lesion boundaries are not clearly defined and the transition

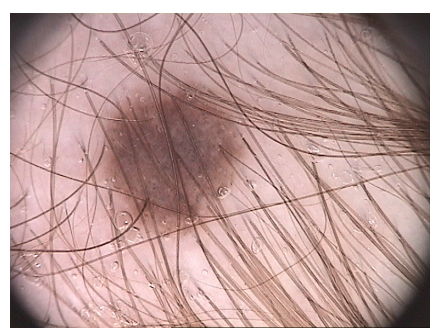

(a) Hair

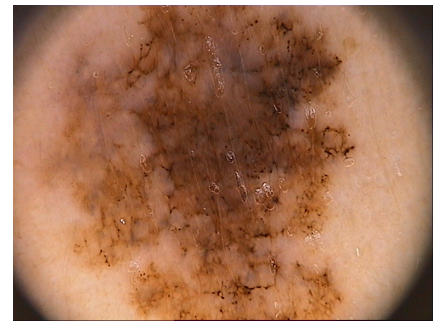

(c) High Texture

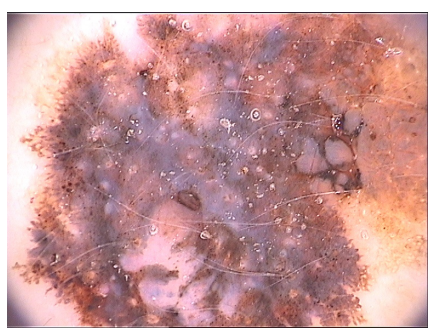

(b) Illumination

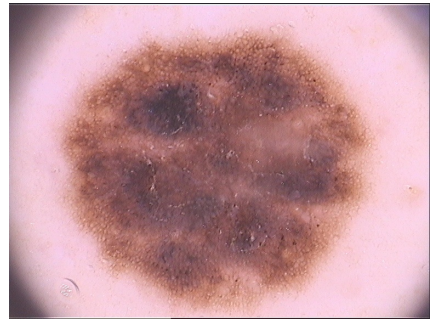

(d) Pigment Network
Fig. 2: The most typical computer vision challenges in dermoscopy images.

from the skin to lesion is smooth. Additionally, there is a significant presence of spurious edges in the images resulting from artifacts such as hair, specular reflections, uneven illumination, or even irregularities resulting from the image texture making segmentation a challenge to handle (Fig. 2).

One class of segmentation methods that has gained significant importance in the recent past is active contours [24], [25]. These are model based segmentation methods that are composed of dynamic fronts which move towards the object boundaries. As the contours approach object boundaries the curve evolution stops, a phenomena that is typically implemented by finding an optimal solution to a minimization problem using the gradient descent algorithm. There can be various criteria to perform the curve evolution that may be based on edges appearing in the images [26] or characteristics of the image regions [27]. These methods can be categorized as either edge based or region based methods depending upon the underlying methodology. However it is important to note that even when an edge based criteria is used, active contours are performing optimization based on a local optimum in the region lying around the curve at a particular location. Thus, the advantages of active contours include their capability to implicitly represent a moving curve such that the regions and the bundaries are captured simultaneously; their robustness against noise that are catered for by the handling of internal forces during curve evolution; and their capability to integrate the desired (custom) properties of the regions. Given this, we have chosen to use active contours to perform the segmentation of lesions in dermoscopy images [10], [28]-[30].

We combine the strengths of thresholding, region and edge characteristics in the images for segmenting dermoscopic lesions using active contours. It is well known that the active contours are based on the evolution of a curve that is initially selected for the algorithm and is thus sensitive to this initialization. The strength of adaptive thresholding is 
utilized in the proposed algorithm by using it to select an initial contour, followed by an optimization framework. This framework is based on maximizing the Kullback-Leibler divergence of gray level distribution between the skin and the lesions. The proposed optimization simplifies to the minimization of the mean shift vectors for both the lesion and the skin with assistance from the image edges. The segmentation results are used to define peripheral regions in the underlying lesions. An empirical study of the significance of lesion periphery has been conducted, quantifying its impact on the identification of melanoma in dermoscopy images.

\section{B. Background on significance of lesion periphery}

Clinical findings pertaining to the significance of the periphery of skin lesion has already been carried out previously. Riccardo et al. [31] found out that all the dermoscopic benign lesions shared a common dermoscopic homogeneous pattern surrounded by peripheral, slightly dotted hyper-pigmentation. Burroni et al. [32] performed a logistic regression analysis validated by a study of statistical significance on different parameters (visual features) affecting the discrimination between in nevi and melanocytic lesions. They concluded that one of the factors, which significantly helps in the discrimination between the said lesions is the presence of pigments clusters at the lesion periphery. These clusters are available in the form of black dots and blotches which help in the identification of melanoma. Henning et al. [33] concluded in their findings that the hyperpigmentation, streaking, radial streaming etc. tend to be focal and asymmetric at the periphery of the melanocytic lesions. These findings are shared by various clinical researchers, emphasizing on the significance of the lesion periphery in differentiating between the normal and benign lesions [32]-[40]. Given this, a quantitative study of the impact of visual features from lesions periphery in comparison to the lesion center can be of significant value towards giving a future direction to the assisted decision systems for diagnostics of skin lesions.

\section{Contributions}

A novel energy functional based on the maximization of distance between the distributions of the object and the background is proposed. This energy functional is embedded as an external energy term in the existing distance regularized level sets evolution (DRLSE) implementation for image segmentation that is composed of the regularization and length terms. We further simplify the formulation and implement it using the finite difference scheme. The proposed formulation is inspired by previous work in [41] where the application was image registration. We quantitatively validate the significance of the peripheral region of the skin lesion as opposed to using the full lesion for characterization purposes. This is done by performing feature extraction and classification on the segmented lesion by selecting peripheral regions of different sizes and performing quantitative validation. The benchmark for this performance is established by performing classification on lesion peripheral regions obtained using manual annotations. It is important to note that the main aim of this paper is on proposing a novel segmentation method followed by an analysis of the contribution of lesion periphery towards classification, and not on improving the overall classification results. Therefore, we use only texture features among all the features defined in the ABCD rules and did not consider the other shape and color based features as the relative conclusions on the lesion periphery analysis will not change.

The overall organization of the paper is as follows: We propose the novel segmentation method (Section III), followed by a description on feature extraction (Section IV). We finally present our experimental results (Section V) and conclude the paper (Section VI).

\section{Segmentation}

\section{A. Background}

We will now explain our modeling assumptions and notations and define our segmentation problem. Let $\Omega$ be the image domain and $I: \Omega \rightarrow \mathbb{R}^{2}$ be a gray level image. This domain has two mutually exclusive subdomains, $\Omega^{-}$and $\Omega^{+}$, indicating the object and background respectively. These subdomains are segregated by a level set function $\phi: \Omega \rightarrow \mathbb{R}^{2}$ that represents the curve indicating the boundary of the region of interest in the image. This function, also known as an active contour is evolved to fit the real boundaries of the objects. The sequence of level set functions for this evolution process can be easily constructed using the variational framework. More specifically, this evolution can be defined using the gradient flow that minimizes an appropriate cost function. Typically in variational level sets [42], this cost function is defined as an energy functional $\mathcal{J}(\phi)$

$$
\mathcal{J}(\phi)=\mu \mathcal{R}(\phi)+\lambda \mathcal{L}(\phi)+\alpha \mathcal{A}(\phi)
$$

where $\mathcal{R}(\phi)$ is the regularization term for the level set function, $\mathcal{L}(\phi)$ is the length term and $\mathcal{A}(\phi)$ is the area term in the level sets framework and $\mu, \lambda$ and $\alpha$ are their respective weighting factors controlling the curve evolution. The regularization and length terms are defined as

$$
\mathcal{R}(\phi)=\frac{1}{2} \int_{\Omega}(\nabla \phi-1)^{2} d \Omega
$$

which ensures that the evolving curve stays a signed distance function, and

$$
\mathcal{L}(\phi)=\int_{\Omega} \delta(\phi)|\nabla \phi| d \Omega
$$

which represents the contour length which needs to be minimal to guarantee smooth convergence

$$
\frac{\partial \phi}{\partial t}=-\frac{\partial \mathcal{J}}{\partial \phi}
$$

Given this, the first variation of $\mathcal{J}$ can be written as

$$
\frac{\partial \mathcal{J}}{\partial \phi}=\mu(\delta(\phi)-k)+\lambda k+\alpha \frac{\partial A(\phi)}{\partial \phi}
$$

where $k=\nabla \cdot \frac{\nabla \phi}{|\nabla \phi|}$ is the curvature of the evolving front [27] and $\delta$ is the derivative of the Heaveside function. 
We adapt the regularization (eq. 2) and length (eq. 3) terms fully from the DRLSE implementation whereas we propose a novel area term based on the external energy, $\mathcal{A}(\phi)$ for curve evolution.

\section{B. Problem Formulation}

Let us assume that an image is composed of two mutually exclusive regions: the object and the background, and these regions can be represented by their respective distributions. Given that we can extract some probability distributions that have the ability to characterize the lesion and the skin distinctively, a robust criteria for segmentation is to maximize the distance between the distributions of the object and the background using a relevant distance measure. In this paper, we have used the Kullback-Leibler (KL) divergence for this purpose. This choice is motivated by the fact that the KLdivergence is a non-negative measure and is zero if the subject distributions are the same. This behavior of the KL-divergence is unique given that the other measure such as Bhattacharya distance is non-zero even if the distributions are equal [43], [44]. The shortcoming of KL-divergence is that it is not a true distance measure as it does not satisfy triangle inequality. To make this measure symmetric, we define $A(\phi)$

$$
\mathcal{A}(\phi)=D\left\{P\left(\Omega^{-}\right) \| P\left(\Omega^{+}\right)\right\}+D\left\{P\left(\Omega^{+}\right) \| P\left(\Omega^{-}\right)\right\}
$$

where $D\left\{P\left(\Omega^{-}\right) \| P\left(\Omega^{+}\right)\right\}$is the Kullback-Leibler (KL) distance between the probability density estimate of the object $P\left(\Omega^{-}\right)$(lesion) and the background $P\left(\Omega^{+}\right)$and $D\left\{P\left(\Omega^{+}\right) \| P\left(\Omega^{-}\right)\right\}$is similarly defined. It can be noticed that the usage of these two KL divergence terms makes $A(\phi)$ symmetric. The KL divergence between two distributions is defined as follows:

$$
D_{\Omega^{-}}=D\left\{P\left(\Omega^{-}\right) \| P\left(\Omega^{+}\right)\right\}=\int_{\Omega} P\left(\Omega^{-}\right) \log \frac{P\left(\Omega^{-}\right)}{P\left(\Omega^{+}\right)} d \Omega
$$

In order to obtain a good segmentation result, the distance between the lesion and the skin should be maximized. Consequently, the area term for an optimal $\phi$ can be written as:

$$
\mathcal{A}\left(\phi^{*}\right)=\arg \max _{\phi} \mathcal{A}(\phi)
$$

where $\phi^{*}$ is the optimal $\phi$ that maximizes $A(\phi)$. This maximization can be conveniently converted to the following minimization problem:

$$
\begin{aligned}
\mathcal{A}\left(\phi^{*}\right) & =\arg \min _{\phi}-\mathcal{A}(\phi) \\
& =\arg \min _{\phi}\left\{-D_{\Omega^{-}}-D_{\Omega^{+}}\right\}
\end{aligned}
$$

Rather than solving the problem posed in equation 9 directly (which is usually impossible), the search for a $\phi$ that proves to be a minimizer for $\mathcal{J}$ is done using the "gradient descent" strategy. Assuming that $\mathcal{A}$ is sufficiently regular, its first variation at $\phi \in \mathcal{F}$ in the direction $\nabla \phi \in \mathcal{F}$ is defined as

$$
\delta \mathcal{A}(\phi)=\lim _{\epsilon \rightarrow 0} \frac{\mathcal{A}(\phi+\epsilon \nabla \phi)-\phi}{\epsilon}=\left.\frac{d \mathcal{A}(\phi+\epsilon \nabla \phi)}{d \epsilon}\right|_{\epsilon=0}
$$

If a minimizer $\phi^{*}$ of $\mathcal{A}$ exists, then $\delta \mathcal{A}\left(\phi^{*}, \nabla \phi\right)=0$ must hold for every $\nabla \phi \in \mathcal{F}$ known as the Euler-Lagrange equation, where $\nabla \phi=\left[\begin{array}{ll}\partial \phi / \partial x & \partial \phi / \partial y\end{array}\right]$ indicates the direction of curve evolution. Assuming that $\mathcal{F}$ is a linear subspace of a Hilbert subspace $H$, with a scalar product $\langle., .\rangle_{H}$, we define the gradient $\nabla \mathcal{A}$ by requiring

$$
\delta \mathcal{A}=\langle\nabla \mathcal{A}, \nabla \phi\rangle_{H}
$$

where $\nabla \mathcal{A}$ can be calculated using the gradient of equation 9 ,

$$
\nabla \mathcal{A}(\phi)=-\nabla D_{\Omega^{-}}-\nabla D_{\Omega^{+}}
$$

Equation 12 forms the basis of the optimization problem posed in this paper. It is the external energy term in the objective function and is obtained from the image data.

\section{Proposed Optimization}

Let us consider equation 7

$$
\begin{aligned}
D_{\Omega^{-}}^{*}=-D_{\Omega^{-}}=- & \int_{\Omega} P_{\phi}\left(\Omega^{-}\right) \log \frac{P_{\phi}\left(\Omega^{-}\right)}{P_{\phi}\left(\Omega^{+}\right)} d \Omega \\
& =\int_{\Omega} P_{\phi}\left(\Omega^{-}\right) \log \frac{P_{\phi}\left(\Omega^{+}\right)}{P_{\phi}\left(\Omega^{-}\right)} d \Omega
\end{aligned}
$$

An explicit computation of the first variation of $D_{\Omega^{-}}^{*}$ yields (see the Appendix):

$$
\delta D_{\Omega^{-}}^{*}=-\int_{\Omega^{-}} \delta P\left(\Omega^{-}\right) \log P\left(\Omega^{-}\right) d \Omega^{-}
$$

where $\delta P\left(\Omega^{-}\right)$is the partial derivative of $P\left(\Omega^{-}\right)$. It is well known that the kernel density estimation can be used for the calculation of $P\left(\Omega^{-}\right)$as follows

$$
P\left(\Omega^{-}\right)=\frac{1}{\left|\Omega^{-}\right|} \int_{\Omega^{-}} G(I(x)-i) d x
$$

where $\left|\Omega^{-}\right|$indicates the area of the inner region of the evolving level sets curve and $G(i)$ is the normalized Gaussian kernel, the first variation of the density estimate can be calculated as follows [41]

$$
\delta P\left(\Omega^{-}\right)=\frac{1}{\left|\Omega^{-}\right|} \int_{\Omega^{-}} \partial G(I(x)-i) \nabla I . \nabla \phi d x .
$$

Substituting equation 15 into equation 14 , we obtain

$\delta D_{\Omega^{-}}^{*}=\frac{-1}{\left|\Omega^{-}\right|} \int_{\Omega^{-}} \int_{\Omega^{-}} \log P\left(\Omega^{-}\right) \partial G(I-i) \nabla I . \nabla \phi d x d \Omega^{-}$.

A convolution with respect to $i$ appears in this expression. It commutes with the derivative operator giving us

$$
\delta D_{\Omega^{-}}^{*}=\frac{-1}{\left|\Omega^{-}\right|} \int_{\Omega^{-}}\left(\partial \log P\left(\Omega^{-}\right) \star G\right) \nabla I . \nabla \phi d x .
$$


Let us now define

$$
L=\frac{1}{\left|\Omega^{-}\right|} G \star \partial \log P\left(\Omega^{-}\right)=\frac{1}{\left|\Omega^{-}\right|} G \star \frac{\partial P_{\phi}\left(\Omega^{-}\right)}{P_{\phi}\left(\Omega^{-}\right)}
$$

Substituting equation 17 in equation 16, we obtain

$$
\delta D_{\Omega^{-}}^{*}=-\int_{\Omega^{-}} L \nabla I . \nabla \phi d x .
$$

By identifying this expression as a scalar product in $L^{2}$ by similarity with equation 11 we define $\nabla D_{\Omega^{-}}^{*}$ as follows

$$
\delta D_{\Omega^{-}}^{*}=\left\langle\nabla D_{\Omega^{-}}^{*}, \nabla \phi\right\rangle_{2} .
$$

Comparing equation 19 and 18, we can deduce that

$$
\nabla D_{\Omega^{-}}^{*}=-L \nabla I
$$

Making substitutions from equations 17 and 18 into equation 20 we obtain,

$$
\nabla D_{\Omega^{-}}^{*}=\frac{-1}{\left|\Omega^{-}\right|} \underbrace{\left(G \star \frac{\partial P_{\phi}\left(\Omega^{-}\right)}{P_{\phi}\left(\Omega^{-}\right)}\right)}_{=0 \text { at steady state }} \nabla I
$$

where $m\left(\Omega^{-}\right)=\partial P_{\phi}\left(\Omega^{-}\right) / P_{\phi}\left(\Omega^{-}\right)$forms the basis of optimization for this term. The term $\nabla D_{\Omega^{-}}^{*}$ tries to move the intensity of $\Omega^{-}$closer to its local maximum (lesion). A similar term for $\nabla D_{\Omega^{+}}^{*}$ can be written as:

$$
\nabla D_{\Omega^{+}}^{*}=\frac{-1}{\left|\Omega^{+}\right|} \underbrace{\left(G \star \frac{\partial P_{\phi}\left(\Omega^{+}\right)}{P_{\phi}\left(\Omega^{+}\right)}\right)}_{=0 \text { at steady state }} \nabla I
$$

where $m\left(\Omega^{+}\right)=\partial P_{\phi}\left(\Omega^{+}\right) / P_{\phi}\left(\Omega^{+}\right)$is the basis of optimization for this term and to tries to move the intensity of $\Omega^{+}$ closer to its local maximum (background). The optimization problem posed in equation 12 can be solved by minimizing

$$
\nabla A=-G \star\left\{m\left(\Omega^{+}\right)+m\left(\Omega^{-}\right)\right\}
$$

The complete optimization problem after incorporating the regularization and length terms can be written as:

$$
\nabla J=\mu(\delta(\phi)-k)+\lambda k+\alpha G \star\left\{m\left(\Omega^{+}\right)+m\left(\Omega^{-}\right)\right\}
$$

\section{Implementation}

In our specific scenario, we are performing the segmentation of dermoscopy images. We are using the distribution of minimum of three channels in RGB (minRGB) of the lesion and skin for the purpose of segmentation, due to its effectiveness in dermoscopy [45]. An initial curve is selected using adaptive thresholding [10]. For the implementation of regularization and length terms, we are employing Li's [42] implementation. The area term is composed of two important variables, $m\left(\Omega^{+}\right)$ and $m\left(\Omega^{-}\right)$which indicate the modes of a mean shift process, that iteratively converge to their respective local maxima at the end of the iterative process [41]. There are four parameters in the proposed model i.e., $\lambda, \mu, \alpha$ and $\nabla t$ (time step). The model is adapted from DRLSE which is not sensitive to the choice of these parameters [42]. However, these parameters are fixed as $\lambda=5.0, \mu=0.04, \alpha=1.0$ and $\nabla t=5.0$. The curve evolution process in the proposed model will attain a steady state when the mean shift of the density estimate is at a steady state for both the lesion and the background.

\section{FEATURE EXTRACTION}

After segmentation of the images, we extract the peripheral region of the lesion followed by feature extraction using local binary patterns.

\section{A. Periphery Extraction}

Clinical findings have concluded that the visual characteristics of the lesion that conform to the presence or absence of melanoma in dermoscopy images lie on the periphery region of the lesion. These regions are populated with two pronounced dermoscopic structures in case of melanoma: pigment networks and streaks. A pigment network is considered as one of the key dermoscopic structures that appears due to the existence of melanin in deep layers of the skin. Therefore, the pigment network is considered as a hallmark of melanocytic lesion. This structure has a very specific shape that visually appears as a grid of thin dark lines appearning on a lighter background forming a pattern called reticular, resembling a honey comb. The pigment network is more pronounced at the periphery of lesions and usually ends abruptly at the end of the lesion. The streaks are also important visual features in dermoscopy images that can considered interchangeably with with radial streaming or pseudopods because of the same histopathological correlation [46]. Radial streaming is a linear extension of pigment at the periphery of a lesion as radially arranged linear structures in the lesion growth direction, and pseudopods represent finger-like projections of dark pigment (brown to black) at the periphery of the lesion [46].

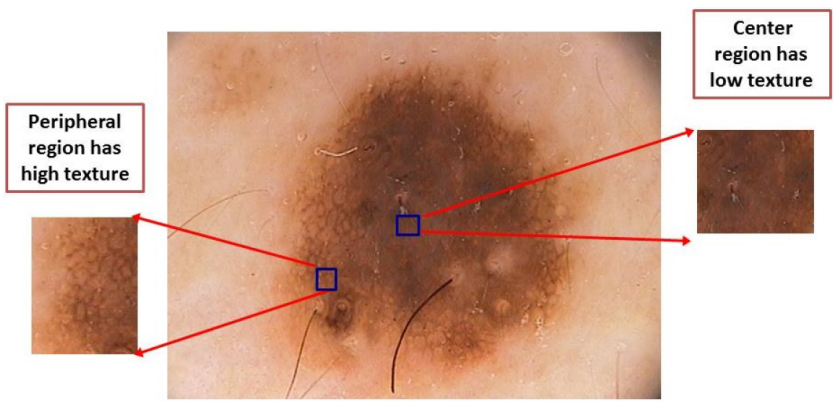

Fig. 3: Texture in the periphery and center of the skin lesion.

Given that the periphery of the lesion in dermoscopy images exhibits significant information regarding the clinical diagnostics of the skin, we are curious if the texture features obtained only from the image regions in the lesion periphery are more discriminant than the more conventional full region descriptors. 


\section{B. Texture features}

As discussed in Section IV, dermoscopy allows the identification of dozens of morphological features such as pigment networks, dots/globules, streaks, blotches etc. All these features are basically differential structures which are strong indicators of melanoma [12]. Any computer vision algorithm that has to be used for assisted diagnostic support for dermoscopy images should have the ability to quantify these differential structures. Local binary patterns (LBP) is a texture feature extraction methodology that can extract local image characteristics such as blobs, spots, corners and edges based on a very local analysis of the images. Given this, our natural choice for feature extraction from dermoscopy images is LBP.

1) Local Binary Patterns (LBP): The LBP is a gray scale invariant operator that was proposed initially by Ojala et al. [47]. The method is based on generating a binary pattern at a pixel by calculating the difference of the center pixel from its neighbors followed by binarizing the differences and concatenating the results in a clockwise way to form a decimal number representing the underlying pattern at a pixel.

$$
L B P_{P, R}=\sum_{p=0}^{P-1} s\left(g_{p}-g_{c}\right) 2^{p}, s(x)= \begin{cases}1 & x \leq 0 \\ 0 & x>0\end{cases}
$$

where $g_{c}$ and $g_{p}$ denote the gray level values of the central pixel and its neighbor respectively, and $p$ is the index of the neighbor. $P$ is the number of the neighbors in a circular set surrounding a pixel at a radius of $R$ from $g_{c}$. Suppose that the coordinate $g_{c}$ is $(0,0)$, the coordinate of each neighboring pixel $g_{p}$ is determined according to its index $p$ and parameter $(P, R)$ as $(R \cos (2 \pi p / P), R \sin (2 \pi p / P))$. The gray values of the neighbors that are not located at the image grids can be estimated by an interpolation operator. Uniformity of an LBP can be quantified as

$$
\begin{array}{r}
U\left(L B P_{P, R}\right)=\left|s\left(g_{P-1}-g_{c}\right)-s\left(g_{0}-g_{c}\right)\right|+ \\
\sum_{p=1}^{P-1}\left|s\left(g_{p}-g_{c}\right)-s\left(g_{p-1}-g_{c}\right)\right|
\end{array}
$$

which corresponds to the number of spatial transitions (bitwise $0 / 1$ changes) in a pattern. An LBP with a uniformity measure of at most 2 is taken as a uniform LBP. The motivation for using uniform LBPs is their ability to detect the important intrinsic characteristics of textures like spots, line edges, edges and corners (Fig. 4).

2) Joint histogram of multiresolution LBP and contrast: Although traditional LBPs capture the texture patterns very efficiently, the description of texture content can be enhanced if the strength of LBPs is also taken into account. This is mainly because the perception of visual textures is mainly created by the variations of contrast in the images and in our specific scenario, the pigment networks and streaks in the images present a stronger contrast. Therefore, we use aim to perform feature extraction from the images using a joint histogram of uniform LBPs and local contrast, where the contrast is calculated as

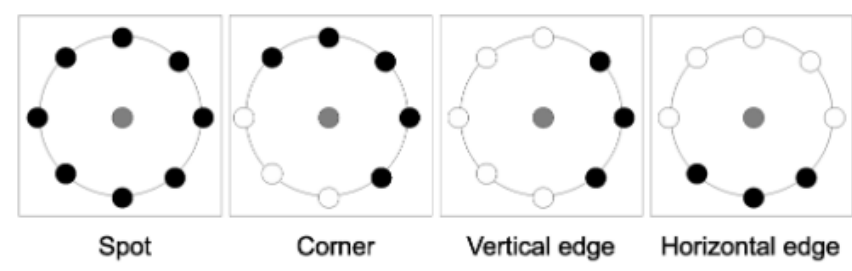

Fig. 4: Examples of different microstructures which are detected using LBP. The pixel in gray color indicates the center pixel gc, the white pixels indicate the neighbors which are greater than $g_{c}$ whereas the black pixels indicate the neighbors which are less than $g_{c}$ (adapted from [48]).

$$
C\left(g_{c}\right)=\sum_{i=0}^{P-1}\left|g_{p}-g_{c}\right|
$$

We calculate LBPs (by selecting the values $(P, R)=\{(8,1),(8,1.5),(8,2)\}$ followed by selecting LBP for the scale (i.e., R) at which we have the highest contrast, indicating a stronger texture component. Joint histograms of the selected LBPs and local contrast are used as image features that are used for the classification of dermoscopy images [49].

\section{EXPERIMENTS}

\section{A. Dataset}

We have used two different datasets for the evaluation of the proposed method:

- The public $\mathrm{PH}^{2}$ dataset [50] that we have used is composed of 200 dermoscopy images with the following composition: $80 \%$ (160) nevus and 20\% (40) melanoma. The images were acquired at Hospital Pedro Hispano, Matosinhos. All images have been acquired during clinical exams using a dermoscope. Each image was manually segmented to identify the lesions and classified by an experienced dermatologist as being normal, atypical nevus (benign) or melanoma (malignant). For our experiments, we perform automatic segmentation of the dermoscopy images and compare the segmentation results against the ground truth which are provided to us by the physicians.

- The second dataset that we have used for evaluation is the International Skin Imaging Collaboration (ISIC) public dataset [51] which contain about 10,000 dermoscopic images which have been acquired at various international hospitals from different devices. A new dermoscopy imaging segmentation challenge was hosted by ISIC in International Symposium on Biomedical Imaging (ISBI) 2016. For carrying out our study, we used 900 dermoscopic images used in this challenge. This is a challenging dataset with various visual artifacts such as color, hair, calibration chart ruler etc. The dataset has been manually segmented by expert physicians.

Manual annotations of both the datasets by expert dermatologists have been made available as ground truth for evaluation purposes. 


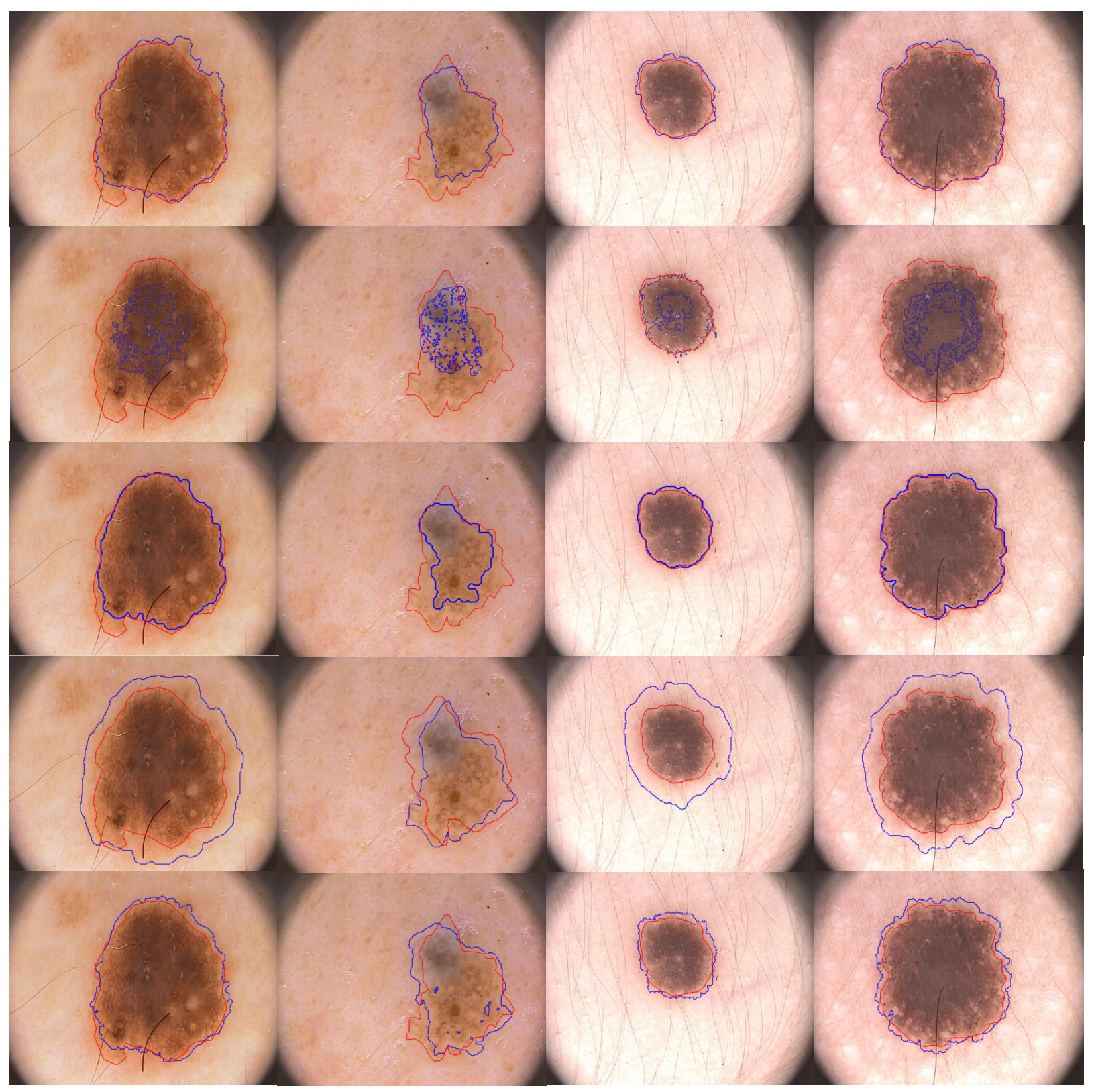

Fig. 5: Some example images segmented using various segmentation methods: DRLSE (row 1, blue contour), LRSI (row 2, blue contour), C-LS (row 3, blue contour), ACWC (row 4, blue contour) and KL-LS (row 5, blue contour). The red contours indicate manual annotations done by the physicians.

\section{B. Segmentation Results}

1) Experimental Setup and Implementation Details: We have compared the proposed method (Kullback-Leibler based level sets, KL-LS) with four other segmentation methods that are based on active contours: Chen based level sets (CLS) [10], the distance regularized level sets (DRLSE) [42], level sets robust to intensity inhomogenity (LSRI) [52] and level sets with creases (ACWC) [53]. We applied hair removal as a preprocessing step for both the $\mathrm{PH}^{2}$ and ISIC datasets using the algorithm proposed by Lee et al [54]. The following setting was used for the segmentation methods: In all the above mentioned methods, an initial seed segmentation is required for which we have used adaptive thresholding (AT) [10], except C-LS for which the results obtained by Euijoon et al. [55] and [56] were directly used. We applied the basic post processing such as region filling (dark pixels completely surrounded by brighter pixels) and noise removal (isolated group of pixels) to all the methods.

2) Performance metrics: We have used three standard metrics that have been used by various researchers for evaluation of the segmentation results: The Dice similarity coefficient that is defined as follows: [57].

$$
D S C(A, S)=\frac{2 N(A \cap S)}{N(A)+N(S)}
$$

where $A$ is the ground truth and $S$ is the segmentation 


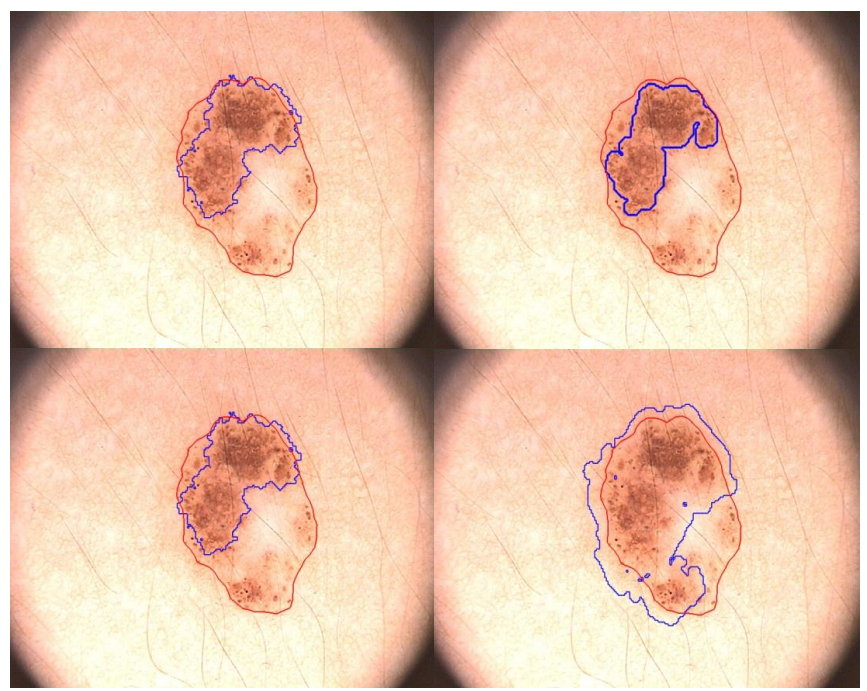

Fig. 6: Segmentation of split lesion: DRLSE (top left, blue contour), C-LS (top right, blue contour), ACWC (bottom left, blue contour) and KL-LS (bottom right, blue contour). The red contours indicate manual annotations done by the physicians.

result. The DSC values lie between 0 and 1 for zero overlap and identical contours respectively. The Hammoude distance (HM) and XOR measure the dissimilarity between the result of segmentation and ground truth, defined as:

$$
\begin{gathered}
H M(A, S)=\frac{N(A \cup S)-N(A \cap S)}{N(A \cup S)} \\
X O R(A, S)=\frac{N(A \cup S)-N(A \cap S)}{N(S)}
\end{gathered}
$$

A higher magnitude of both these measures indicates a higher degree of dissimilarity between $A$ and $S$ and vice versa.

3) Evaluation of $\mathrm{PH}^{2}$ dataset: Our experiments show that in general, all active contours based methods show good segmentation results. Therefore, generally they are useful for the design of assisted diagnostic systems for dermoscopy. The proposed method (KL-LS) obtains very good segmentation results (Table I). It obtain the best overall DSC value as compared to the other methods. In comparison, the worst segmentation results were yielded by ACWC due to a very specific reason: the image smoothing process in multilocal creasness operator used in ACWC significantly pronounces the boundary around the lesions. Effectively the contours encapsulate a slightly larger region around the lesions, generating segmentations having an offset consistently across the whole dataset effecting the DSC values for all images. Although the overall DSC is poor for ACWC, the overall HM and XOR values are very good because the encapsulation of larger regions increases the number of pixels in the segments increasing the $N(A \cup S)$ and $N(S)$ values thus decreasing the values of HM and XOR respectively (fourth row in Fig. 5). In general, C-LS is most comparable to KL-LS showing good segmentation results.
TABLE I: Segmentation results measured by DSC, HM and XOR averaged over 200 images of the $\mathrm{PH}^{2}$ dataset.

\begin{tabular}{cccccc}
\hline \hline Mean & DRLSE & LRSI & C-LS & ACWC & KL-LS \\
\hline DSC & 82.17 & 80.53 & 84.82 & 75.95 & $\mathbf{8 6 . 5 4}$ \\
HM & 28.38 & 47.45 & 24.58 & $\mathbf{2 0 . 4 7}$ & 23.01 \\
XOR & 49.39 & 49.51 & 28.93 & $\mathbf{2 2 . 3 3}$ & 25.14 \\
\hline \hline
\end{tabular}

TABLE II: Segmentation results measured by DSC, HM and XOR averaged over 900 images of the ISIC dataset.

\begin{tabular}{cccccc}
\hline \hline Mean & DRLSE & LRSI & C-LS & ACWC & KL-LS \\
\hline DSC & 74.81 & 73.11 & 79.61 & 65.85 & $\mathbf{8 0 . 1 7}$ \\
HM & 29.57 & 35.44 & 30.00 & $\mathbf{2 2 . 1 0}$ & 25.25 \\
XOR & 45.65 & 48.36 & 50.58 & $\mathbf{2 4 . 5 9}$ & 29.38 \\
\hline \hline
\end{tabular}

4) Evaluation of ISIC dataset: The ISIC is a more challenging dataset having more visual artifacts such as presence of hair, presence of calibration scale and a circular mask appearing in the border of some images. Also, there is a complex variation in lesion locations, lighting conditions and nonuniform vignetting [58]. Therefore, all the methods yield relatively low segmentation results as compared to those for the $\mathrm{PH}^{2}$ dataset. However, KL-LS outperforms the other methods that have been considered in this paper (Table II).

5) Comparison With Saliency Detection Methods: One class of segmentation methods that has gained significant importance in the recent times is the saliency detection methods. Although the methods are unsupervised, background and foreground templates from some images which can represent good examples of the skin and lesion respectively are used to learn the structural models represented by them inducing prior knowledge to the segmentation models [55]. Accordingly, we have included some saliency based methods in our comparison to study the effects of adding some prior knowledge to the segmentation methods. The results for two different methods have been used for comparison: saliency based skin lesion segmentation (SSLS) [55] and robust SSLS (RSSLS) [59], which show the best results on the datasets considered in this paper. The results for these methods have been quoted directly from [59]. On the $\mathrm{PH}^{2}$ dataset, RSSLS shows better performance using all metrics. On the contrary, KL-LS performs better using two metrics (HM and XOR) on the ISIC dataset. It should be noted that the results obtained on ISIC exhibit a much higher statistical significance as the size of this dataset is about 4.5 times that of the $\mathrm{PH}^{2}$ dataset. Also, the gap in DSC narrows from $4.51 \%$ for $\mathrm{PH}^{2}$ to about $3.24 \%$ for ISIC. This analysis brings us to another interesting conclusion: adding some prior knowledge to the segmentation methods results in an improved performance. The proposed KL-LS is an unsupervised segmentation method in which the curve evolution is purely based on the fact that the final segmentation results should maximize the difference between the lesion and the background (as assumed in many other methods such as [60]). Although the KL-LS does not present optimal (best) segmentation results (specifically for $\mathrm{PH}^{2}$ dataset), the ability of active contours to incorporate the custom features of specific 
imaging scenarios makes it a very suitable contender for segmentation in specific scenarios such as dermoscopy.

TABLE III: Segmentation results measured by DSC, HM and XOR averaged $200 \mathrm{PH}^{2}$ and 900 ISIC images.

\begin{tabular}{cccc}
\hline \hline Mean & SSLS & RSSLS & KL-LS \\
\hline DSC & 85.34 & $\mathbf{9 1 . 0 5}$ & 86.54 \\
DSC $^{2}$ & 80.61 & $\mathbf{8 3 . 4 1}$ & 80.17 \\
HM $^{1}$ & 22.92 & $\mathbf{1 5 . 4 9}$ & 23.01 \\
HM $^{2}$ & 29.78 & 25.67 & $\mathbf{2 5 . 2 5}$ \\
XOR $^{1}$ & 25.27 & $\mathbf{1 6 . 4 5}$ & 25.14 \\
XOR $^{2}$ & 38.40 & 36.21 & $\mathbf{2 9 . 3 8}$ \\
\hline
\end{tabular}

${ }^{1}$ Images from $\mathrm{PH}^{2}$ dataset.

${ }^{2}$ Images from ISIC dataset.

\section{Classification results}

The objective of the classification task in this paper is to quantify the relative contribution of lesion periphery and its size on the identification of melanoma, in contrast to using image description from full lesion. In this context, the lesion periphery is defined as a region that starts with the boundaries of the segmented lesion and grows inward (towards the lesion center). We have started with a small periphery (5 pixels) and varied its size upto 45 pixels to assess the effects of increasing the periphery size on the classification results of the lesion (Fig. 7). We have performed our classification results on the Weka data mining tool using 10 -fold cross validation using two different classifiers for classification purposes i.e., one-nearest neighbor (1NN) and support vector machine (SVM) with linear kernel. This choice is complementary and is motivated by two important considerations: the $1 \mathrm{NN}$ classifier will help us conceive if the lesion is linearly separable from the skin or not whereas SVM is a more generic classifier that seeks to obtain the margins that maximize the class separation. Additionally, we want to analyze the trend adopted by different classifiers for the size of periphery to ensure that the results are not classifier specific and are generic enough to make robust conclusions. For feature extraction, we have used the standard uniform local binary patterns (LBP) which yield 60 features and joint histogram of local binary patterns and local contrast LBP_C (120 features: 60 features for uniform LBP
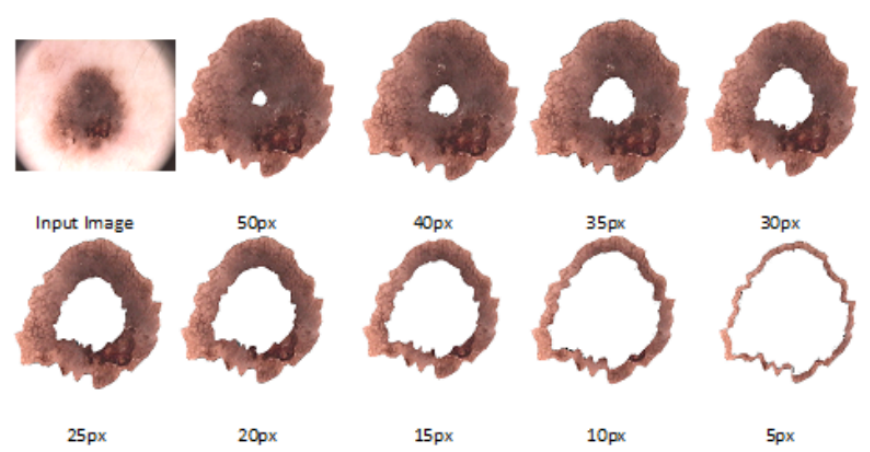

$5 p x$

and 2 features for contrast i.e., low or high). Although, the researchers have already found that LBP_C outperforms LBP for feature extraction from dermoscopy images, the main objective is to evaluate the trend of changing the lesion periphery on the classification of lesion and not on obtaining the 'best' classification results. These are histogram based features and thus the number of features stay the same irrespective of the size of the peripheral region of the lesion.

Our experiments demonstrate that better prospects of identifying melanoma exist when only the lesion periphery is used for classification purposes. This conclusion is consistent when manual annotations and the proposed method are used. The general trend is that as we increase the periphery size, the classification results start to improve until we reach a periphery of 35 pixels width at the lesion periphery. When the size increases beyond 35 pixels, the classification results start to depreciate (Table IV and V). This observation is consistent for the two different classifiers and feature extraction methodologies that have been considered in this paper. We attribute this result to the fact that the peripheral region of the lesions is mainly exhibiting the differential structures which are present in the lesion, which mainly include the pigment network and streaks. These particular characteristics of the lesion are more relevant in identifying melanoma. When full lesion is used for feature extraction, some redundancy is added to the descriptor which is contributing to the lower classification accuracies in identifying melanoma. It is important to note that the underlying conclusions are consistent with the clinical findings that the lesion periphery is more significant for identifying melanoma in dermoscopy images.

\section{CONCLUSION}

This paper deals with a study of assisted diagnostics applied to dermoscopy images. We have focused on proposing a novel image segmentation algorithm followed by a study of the significance of periphery in characterizing skin lesions, in contrast to using the full lesion. For segmentation, we have proposed an optimization problem where the objective function is to maximize the distance of gray level distribution between the skin and the lesion. The proposed method exploits advantages from the different categories of segmentation methods including thresholding, edge based and region based methods given that we use adaptive thresholding for contour initialization, followed by the proposed optimization criteria which uses a region based term (convergence of the means of the lesion and skin to their local maxima) integrated with the image gradient (edges) to perform image segmentation. Very good segmentation results are obtained as the proposed method exhibits various strengths which are usually hard to find in one segmentation method. The proposed method is based on the maximization of KL distance between the distributions of lesion and the background, which is a standard segmentation optimization problem. There is a lack of context aware information (specific texture, color, shape etc. features) in the proposed method. Very recently, methods which incorporate prior knowledge have been used such as SSLS and RSSLS which show very good segmentation results on dermoscopy

Fig. 7: Texture in the periphery and center of the skin lesion. 
TABLE IV: Classification results obtained as the periphery of the lesion varies from 5 pixels to 45 pixels on $\mathrm{PH}^{2}$ dataset using ground truth (Manual) and segmentation (Automatic). The periphery is obtained from the segmentation results that are obtained using KL-LS (LBP - Local Binary Patterns; LBP_C - Joint histogram of LBP and local contrast).

\begin{tabular}{|c|c|c|c|c|c|c|c|c|c|c|c|c|}
\hline \multirow[t]{2}{*}{ Class } & \multirow[t]{2}{*}{ Segmentation } & \multirow[t]{2}{*}{ Method } & \multicolumn{2}{|c|}{$5 p x$} & \multicolumn{2}{|c|}{$15 p x$} & \multicolumn{2}{|c|}{$25 p x$} & \multicolumn{2}{|c|}{ 35px } & \multicolumn{2}{|c|}{$45 p x$} \\
\hline & & & Acc \% & ROC \% & Acc \% & ROC \% & Acc \% & ROC \% & Acc \% & ROC \% & Acc \% & ROC $\%$ \\
\hline \multirow{5}{*}{ SVM } & \multirow{2}{*}{ Manual } & LBP & 84.9 & 74.6 & 85.4 & 79.8 & 89.7 & 83.9 & 90.4 & 85.1 & 83 & 80.6 \\
\hline & & LBP_C & 87.65 & 83.1 & 90.85 & 86.2 & 91.2 & 86.9 & 90.1 & 85 & 88.75 & 77.6 \\
\hline & & & & & & & & & & & & \\
\hline & \multirow{2}{*}{ Automatic } & LBP & 79.15 & 69.3 & 80.74 & 69.9 & 86 & 74.6 & 89.2 & 75.6 & 84.5 & 76.1 \\
\hline & & LBP_C & 85.5 & 82.2 & 90.45 & 84.9 & 89.02 & 79.8 & 84.9 & 74 & 82.25 & 70.1 \\
\hline \multirow{5}{*}{ KNN } & \multirow{2}{*}{ Manual } & LBP & 77 & 66.1 & 78.8 & 69.8 & 84 & 71.9 & 85.1 & 75.4 & 84.5 & 73.5 \\
\hline & & LBP_C & 76 & 69.8 & 86.4 & 81.2 & 85.4 & 80 & 81.4 & 75.3 & 77 & 57.3 \\
\hline & & & & & & & & & & & & \\
\hline & \multirow{2}{*}{ Automatic } & LBP & 68.1 & 59.4 & 75.8 & 68.6 & 82.13 & 70.4 & 85.01 & 71.8 & 80.4 & 69.2 \\
\hline & & LBP_C & 80.9 & 69.9 & 85.3 & 75 & 87.7 & 80.4 & 83.7 & 71.7 & 74 & 53.6 \\
\hline
\end{tabular}

TABLE V: Classification results obtained as the periphery of the lesion varies from 5 pixels to 45 pixels on ISIC dataset using ground truth (Manual) and segmentation (Automatic). The periphery is obtained from the segmentation results that are obtained using KL-LS (LBP - Local Binary Patterns; LBP_C - Joint histogram of LBP and local contrast).

\begin{tabular}{|c|c|c|c|c|c|c|c|c|c|c|c|c|}
\hline \multirow[t]{2}{*}{ Class } & \multirow[t]{2}{*}{ Segmentation } & \multirow[t]{2}{*}{ Method } & \multicolumn{2}{|c|}{$5 p x$} & \multicolumn{2}{|c|}{$15 p x$} & \multicolumn{2}{|c|}{$25 p x$} & \multicolumn{2}{|c|}{ 35px } & \multicolumn{2}{|c|}{$45 p x$} \\
\hline & & & Acc \% & ROC \% & Acc \% & ROC \% & Acc \% & ROC \% & Acc \% & ROC \% & Acc \% & ROC \% \\
\hline \multirow[b]{2}{*}{ SVM } & Manual & $\begin{array}{c}\text { LBP } \\
\text { LBP_C }\end{array}$ & $\begin{array}{l}63.9 \\
69.2\end{array}$ & $\begin{array}{l}54.2 \\
50.5\end{array}$ & $\begin{array}{l}70.4 \\
72.6\end{array}$ & $\begin{array}{l}68.5 \\
68.8\end{array}$ & $\begin{array}{l}77.6 \\
78.5\end{array}$ & $\begin{array}{l}70.4 \\
71.4\end{array}$ & $\begin{array}{c}84 \\
89.6\end{array}$ & $\begin{array}{l}85.7 \\
87.9\end{array}$ & $\begin{array}{l}80.9 \\
86.9\end{array}$ & $\begin{array}{l}82.1 \\
82.1\end{array}$ \\
\hline & Automatic & $\begin{array}{c}\text { LBP } \\
\text { LBP_C }\end{array}$ & $\begin{array}{l}60.8 \\
64.2 \\
\end{array}$ & $\begin{array}{l}42.1 \\
52.2 \\
\end{array}$ & $\begin{array}{l}68.6 \\
70.4 \\
\end{array}$ & $\begin{array}{l}65.0 \\
62.5 \\
\end{array}$ & $\begin{array}{l}70.4 \\
72.1 \\
\end{array}$ & $\begin{array}{l}61.8 \\
65.6 \\
\end{array}$ & $\begin{array}{l}79.6 \\
79.4 \\
\end{array}$ & $\begin{array}{l}75.2 \\
75.4\end{array}$ & $\begin{array}{l}79.2 \\
80.6\end{array}$ & $\begin{array}{l}74.3 \\
74.7\end{array}$ \\
\hline \multirow{2}{*}{ KNN } & Manual & $\begin{array}{c}\text { LBP } \\
\text { LBP_C }\end{array}$ & $\begin{array}{l}68.6 \\
70.0\end{array}$ & $\begin{array}{l}50.9 \\
69.1\end{array}$ & $\begin{array}{l}69.9 \\
70.7\end{array}$ & $\begin{array}{c}50.7 \\
77\end{array}$ & $\begin{array}{l}75.6 \\
75.9\end{array}$ & $\begin{array}{c}66.6 \\
69\end{array}$ & $\begin{array}{l}77.9 \\
79.8\end{array}$ & $\begin{array}{l}70.2 \\
78.5\end{array}$ & $\begin{array}{l}75.8 \\
70.2\end{array}$ & $\begin{array}{l}70 \\
79\end{array}$ \\
\hline & Automatic & $\begin{array}{c}\text { LBP } \\
\text { LBP_C }\end{array}$ & $\begin{array}{l}66.4 \\
71.5\end{array}$ & $\begin{array}{c}49 \\
70.5\end{array}$ & $\begin{array}{l}72.3 \\
74.8\end{array}$ & $\begin{array}{l}50.1 \\
78.6\end{array}$ & $\begin{array}{l}79.6 \\
80.7\end{array}$ & $\begin{array}{l}75.8 \\
80.9\end{array}$ & $\begin{array}{l}85.1 \\
80.1\end{array}$ & $\begin{array}{l}79.7 \\
82.6\end{array}$ & $\begin{array}{l}84.5 \\
79.7\end{array}$ & $\begin{array}{l}77.9 \\
80.5\end{array}$ \\
\hline
\end{tabular}

images. Given that these methods obtain slightly superior results (adding context awareness gives better results in segmentation) and the fact active contours have the capabilities to incorporate specific features useful in dermoscopy imaging, we believe that there is a reasonable potential of improvement in the proposed method for segmenting dermoscopy images if context awareness is added to the segmentation model.

For assessing the quantitative impact of lesion periphery on the characterization of skin lesions, we have carried out experiments in which after segmentation, instead of using the full lesion, we consider peripheral region of different sizes and study their impact on the classification of skin lesions. Our experiments show that the classification results improve as we increase the periphery size upto about 35 pixels and than the results start to depreciate. The benchmark to validate this fact has been obtained using manual annotations followed by using automatic segmentation, completing the chain of a standard pattern recognition system for assisted diagnosis of dermoscopy images. The relative conclusion that the peripheral features give better results as opposed to using full lesion do not change. This is consistent with the clinical findings that the skin lesions exhibit a border region having dermosopic structures which have a higher significance in the identification of melanoma.
In the future, we intend to work on novel deformable models for segmentation by inducing prior knowledge to the segmentation models. Also, we intend to focus on improving the classification results for the detection of melanoma. For this purpose, we will work on using more adequate visual descriptors that have more physiological importance in identifying melanoma.

\section{APPENDIX}

Let us suppose we have

$$
\begin{aligned}
D & =\int P\left(\Omega^{-}\right) \log \frac{P\left(\Omega^{+}\right)}{P\left(\Omega^{-}\right)} d \Omega \\
& =\int P\left(\Omega^{-}\right)\left[\log P\left(\Omega^{+}\right)-\log P\left(\Omega^{-}\right)\right] d \Omega \\
& =\int P\left(\Omega^{-}\right) \log P\left(\Omega^{+}\right) d \Omega-\int P\left(\Omega^{-}\right) \log P\left(\Omega^{-}\right) d \Omega
\end{aligned}
$$

We will simplify this equation by considering the left and right term of this equation respectively. Let

$$
\begin{aligned}
L & =\int P\left(\Omega^{-}\right) \log P\left(\Omega^{+}\right) d \Omega \\
R & =\int P\left(\Omega^{-}\right) \log P\left(\Omega^{-}\right) d \Omega .
\end{aligned}
$$


Taking derivative of $L$, we get

$\partial L=\left[\int \frac{P\left(\Omega^{-}\right)}{P\left(\Omega^{+}\right)} \partial P\left(\Omega^{+}\right) d \Omega+\int \log P\left(\Omega^{+}\right) \partial P\left(\Omega^{-}\right) d \Omega\right]$

Rearranging the terms,

$$
\begin{aligned}
\partial L= & \int \frac{\partial P\left(\Omega^{+}\right)}{P\left(\Omega^{+}\right)} \underbrace{\int P\left(\Omega^{-}\right) d \Omega^{-}}_{=1} d \Omega^{+}+ \\
& \int \log P\left(\Omega^{+}\right) \underbrace{\int \partial P\left(\Omega^{-}\right) d \Omega^{-}}_{=0} d \Omega^{+}
\end{aligned}
$$

Therefore,

$$
\partial L=\int \frac{\partial P\left(\Omega^{+}\right)}{P\left(\Omega^{+}\right)} d \Omega^{+}
$$

Now applying $u v$ rule of integration where $u=1 / P\left(\Omega^{+}\right)$ and $v=\partial P\left(\Omega^{+}\right)$, we obtain

$$
\begin{gathered}
\partial L=\frac{1}{P\left(\Omega^{+}\right)} \underbrace{\int \partial P\left(\Omega^{+}\right) d \Omega^{+}}_{=0}+ \\
\int \frac{\partial P\left(\Omega^{+}\right)}{P^{2}\left(\Omega^{+}\right)} \underbrace{\int \partial P\left(\Omega^{+}\right) d \Omega^{+}}_{=0} d \Omega^{+} \\
\Rightarrow \partial L=0
\end{gathered}
$$

Now let us consider the equation 33

$$
R=\int P\left(\Omega^{-}\right) \log P\left(\Omega^{-}\right) d \Omega .
$$

Taking derivative of $R$, we get

$$
\partial R=\partial\left[\int P\left(\Omega^{-}\right) \log P\left(\Omega^{-}\right) d \Omega\right]
$$

Commuting derivative and integral operators and derivative, we get

$$
\begin{aligned}
\partial R & =\int\left[\frac{P\left(\Omega^{-}\right)}{P\left(\Omega^{-}\right)} \partial P\left(\Omega^{-}\right)+\log P\left(\Omega^{-}\right) \partial P\left(\Omega^{-}\right)\right] d \Omega \\
& =\int \partial P\left(\Omega^{-}\right) d \Omega+\int \partial P\left(\Omega^{-}\right) \log P\left(\Omega^{-}\right) d \Omega \\
& =\partial \underbrace{\int P\left(\Omega^{-}\right) d \Omega}_{=0 \text { (constant) }}+\int \partial P\left(\Omega^{-}\right) \log P\left(\Omega^{-}\right) d \Omega \\
\Rightarrow \partial R & =\int \partial P\left(\Omega^{-}\right) \log P\left(\Omega^{-}\right) d \Omega
\end{aligned}
$$

Therefore,

$$
\begin{aligned}
\partial D & =\partial L-\partial R \\
& =-\int \partial P\left(\Omega^{-}\right) \log P\left(\Omega^{-}\right) d \Omega
\end{aligned}
$$

\section{REFERENCES}

[1] https://seer.cancer.gov/statfacts/html/melan.html, 2008. [Online; accessed 10-Mar-2017].

[2] A. C. Society, Cancer Facts \& Figures for Hispanics. American Cancer Society., 2009.

[3] M. Sadeghi, T. K. Lee, D. McLean, H. Lui, and M. S. Atkins, "Detection and analysis of irregular streaks in dermoscopic images of skin lesions," Medical Imaging, IEEE Transactions on, vol. 32, no. 5, pp. 849-861, 2013.

[4] G. Argenziano, H. P. Soyer, S. Chimenti, R. Talamini, R. Corona, F. Sera, M. Binder, L. Cerroni, G. De Rosa, G. Ferrara, et al., "Dermoscopy of pigmented skin lesions: results of a consensus meeting via the internet," Journal of the American Academy of Dermatology, vol. 48, no. 5, pp. 679-693, 2003.

[5] J. Mayer, "Systematic review of the diagnostic accuracy of dermatoscopy in detecting malignant melanoma.," The Medical Journal of Australia, vol. 167, no. 4, pp. 206-210, 1997.

[6] M. Binder, M. Puespoeck-Schwarz, A. Steiner, H. Kittler, M. Muellner, K. Wolff, H. Pehamberger, et al., "Epiluminescence microscopy of small pigmented skin lesions: short-term formal training improves the diagnostic performance of dermatologists," Journal of the American Academy of Dermatology, vol. 36, no. 2, pp. 197-202, 1997.

[7] C. Barata, M. Ruela, M. Francisco, T. Mendonça, and J. S. Marques, "Two systems for the detection of melanomas in dermoscopy images using texture and color features," Systems Journal, IEEE, vol. 8, no. 3, pp. 965-979, 2014.

[8] H. Soyer, G. Argenziano, S. Chimenti, S. Menzies, H. Pehamberger, H. Rabinovitz, W. Stolz, and A. Kopf, "Dermoscopy of pigmented skin lesions. an atlas based on the consensus net meeting on dermoscopy 2000," Milan: Edra, 2001.

[9] H. Ganster, A. Pinz, R. Röhrer, E. Wildling, M. Binder, and H. Kittler, "Automated melanoma recognition," Medical Imaging, IEEE Transactions on, vol. 20, no. 3, pp. 233-239, 2001.

[10] M. Silveira, J. C. Nascimento, J. S. Marques, A. R. Marçal, T. Mendonça, S. Yamauchi, J. Maeda, and J. Rozeira, "Comparison of segmentation methods for melanoma diagnosis in dermoscopy images," Selected Topics in Signal Processing, IEEE Journal of, vol. 3, no. 1, pp. 35-45, 2009.

[11] M. E. Celebi, Q. Wen, H. Iyatomi, K. Shimizu, H. Zhou, and G. Schaefer, "A state-of-the-art survey on lesion border detection in dermoscopy images," Dermoscopy Image Analysis, pp. 97-129, 2015.

[12] M. E. Celebi, H. Iyatomi, G. Schaefer, and W. V. Stoecker, "Lesion border detection in dermoscopy images," Computerized medical imaging and graphics, vol. 33, no. 2, pp. 148-153, 2009.

[13] H. Iyatomi, H. Oka, M. E. Celebi, M. Hashimoto, M. Hagiwara, M. Tanaka, and K. Ogawa, "An improved internet-based melanoma screening system with dermatologist-like tumor area extraction algorithm," Computerized Medical Imaging and Graphics, vol. 32, no. 7, pp. 566-579, 2008.

[14] M. Emre Celebi, Y. Alp Aslandogan, W. V. Stoecker, H. Iyatomi, H. Oka, and X. Chen, "Unsupervised border detection in dermoscopy images," Skin Research and Technology, vol. 13, no. 4, pp. 454-462, 2007.

[15] F. Peruch, F. Bogo, M. Bonazza, V.-M. Cappelleri, and E. Peserico, "Simpler, faster, more accurate melanocytic lesion segmentation through meds," IEEE Transactions on Biomedical Engineering, vol. 61, no. 2, pp. 557-565, 2014.

[16] R. Garnavi, M. Aldeen, M. E. Celebi, G. Varigos, and S. Finch, "Border detection in dermoscopy images using hybrid thresholding on optimized color channels," Computerized Medical Imaging and Graphics, vol. 35, no. 2, pp. 105-115, 2011.

[17] M. Emre Celebi, Q. Wen, S. Hwang, H. Iyatomi, and G. Schaefer, "Lesion border detection in dermoscopy images using ensembles of thresholding methods," Skin Research and Technology, vol. 19, no. 1, 2013.

[18] A. Wong, J. Scharcanski, and P. Fieguth, "Automatic skin lesion segmentation via iterative stochastic region merging," Information Technology in Biomedicine, IEEE Transactions on, vol. 15, no. 6, pp. 929-936, 2011.

[19] R. Kéchichian, H. Gong, M. Revenu, O. Lézoray, and M. Desvignes, "New data model for graph-cut segmentation: application to automatic melanoma delineation," in Image Processing (ICIP), 2014 IEEE International Conference on, pp. 892-896, IEEE, 2014.

[20] H. Wang, X. Chen, R. H. Moss, R. J. Stanley, W. V. Stoecker, M. E. Celebi, T. M. Szalapski, J. M. Malters, J. M. Grichnik, A. A. Marghoob, et al., "Watershed segmentation of dermoscopy images using a watershed technique," Skin Research and Technology, vol. 16, no. 3, pp. 378-384, 2010. 
[21] H. Wang, R. H. Moss, X. Chen, R. J. Stanley, W. V. Stoecker, M. E. Celebi, J. M. Malters, J. M. Grichnik, A. A. Marghoob, H. S. Rabinovitz, et al., "Modified watershed technique and post-processing for segmentation of skin lesions in dermoscopy images," Computerized Medical Imaging and Graphics, vol. 35, no. 2, pp. 116-120, 2011.

[22] A. A. Abbas, W.-H. Tan, and X.-N. Guo, "Combined optimal wavelet filters with morphological watershed transform for the segmentation of dermoscopic skin lesions," in PRICAI 2012: Trends in Artificial Intelligence, pp. 722-727, Springer, 2012.

[23] P. Schmid, "Lesion detection in dermatoscopic images using anisotropic diffusion and morphological flooding," in Image Processing, 1999. ICIP 99. Proceedings. 1999 International Conference on, vol. 3, pp. 449-453, IEEE, 1999

[24] C. Xu, D. L. Pham, and J. L. Prince, "Image segmentation using deformable models," Handbook of medical imaging, vol. 2, pp. 129174, 2000.

[25] V. Caselles, R. Kimmel, G. Sapiro, and A. Bovik, "Geometric active contours for image segmentation," Handbook of Video and Image Processing, pp. 613-627, 2005.

[26] C. Li, C. Xu, C. Gui, and M. D. Fox, "Level set evolution without re-initialization: a new variational formulation," in Computer Vision and Pattern Recognition, 2005. CVPR 2005. IEEE Computer Society Conference on, vol. 1, pp. 430-436, IEEE, 2005.

[27] A. A. Farag, E. Munim, H. E. Abd, and J. H. Graham, "A novel approach for lung nodules segmentation in chest ct using level sets," Image Processing, IEEE Transactions on, vol. 22, no. 12, pp. 52025213, 2013.

[28] M. Mete and N. M. Sirakov, "Lesion detection in demoscopy images with novel density-based and active contour approaches," BMC bioinformatics, vol. 11, no. 6, p. S23, 2010.

[29] H. Zhou, G. Schaefer, M. E. Celebi, F. Lin, and T. Liu, "Gradient vector flow with mean shift for skin lesion segmentation," Computerized Medical Imaging and Graphics, vol. 35, no. 2, pp. 121-127, 2011.

[30] H. Zhou, X. Li, G. Schaefer, M. E. Celebi, and P. Miller, "Mean shift based gradient vector flow for image segmentation," Computer Vision and Image Understanding, vol. 117, no. 9, pp. 1004-1016, 2013.

[31] R. Balestri, L. Rizzoli, G. Rech, and C. R. Girardelli, "Dermoscopy of papular epidermal nevus with skyline basal cell layer," Pediatric dermatology, vol. 34, no. 2, 2017.

[32] M. Burroni, P. Sbano, G. Cevenini, M. Risulo, G. Dell'Eva, P. Barbini, C. Miracco, M. Fimiani, L. Andreassi, and P. Rubegni, "Dysplastic naevus vs. in situ melanoma: digital dermoscopy analysis," British Journal of Dermatology, vol. 152, no. 4, pp. 679-684, 2005.

[33] J. S. Henning, S. W. Dusza, S. Q. Wang, A. A. Marghoob, H. S Rabinovitz, D. Polsky, and A. W. Kopf, "The cash (color, architecture, symmetry, and homogeneity) algorithm for dermoscopy," Journal of the American Academy of Dermatology, vol. 56, no. 1, pp. 45-52, 2007.

[34] M. E. Celebi, H. A. Kingravi, B. Uddin, H. Iyatomi, Y. A. Aslandogan, W. V. Stoecker, and R. H. Moss, "A methodological approach to the classification of dermoscopy images," Computerized Medical Imaging and Graphics, vol. 31, no. 6, pp. 362-373, 2007.

[35] H. A. Almubarak, R. J. Stanley, W. V. Stoecker, and R. H. Moss, "Fuzzy color clustering for melanoma diagnosis in dermoscopy images," Information, vol. 8, no. 3, p. 89, 2017.

[36] V. Giorgi, A. Gori, I. Savarese, A. D’errico, M. Grazzini, F. Papi, V. Maio, P. Covarelli, C. Urso, and D. Massi, "Teledermoscopy in doubtful melanocytic lesions: is it really useful?," International journal of dermatology, vol. 55, no. 10, pp. 1119-1123, 2016.

[37] A. Borghi, M. Corazza, S. Minghetti, E. Bianchini, and A. Virgili, "Dermoscopic features of vulvar lichen sclerosus in the setting of a prospective cohort of patients: new observations," Dermatology, vol. 232 , no. 1 , pp. 71-77, 2016

[38] J.-H. Mun, J. Ohn, W.-I. Kim, S.-M. Park, and M.-B. Kim, "Dermoscopy of melanomas on the trunk and extremities in asians," PloS one, vol. 11, no. 7, p. e0158374, 2016.

[39] A. Scope, M. A. Marchetti, A. A. Marghoob, S. W. Dusza, A. C. Geller, J. M. Satagopan, M. A. Weinstock, M. Berwick, and A. C. Halpern, "The study of nevi in children: Principles learned and implications for melanoma diagnosis," Journal of the American Academy of Dermatology, vol. 75, no. 4, pp. 813-823, 2016

[40] Z. J. Wolner, O. Yélamos, K. Liopyris, T. Rogers, M. A. Marchetti, and A. A. Marghoob, "Enhancing skin cancer diagnosis with dermoscopy," Dermatologic Clinics, 2017.
[41] G. H. Valadez, Variational methods for multimodal image matching. $\mathrm{PhD}$ thesis, Université Nice Sophia Antipolis, 2002.

[42] C. Li, C. Xu, C. Gui, and M. D. Fox, "Distance regularized level set evolution and its application to image segmentation," IEEE transactions on image processing, vol. 19, no. 12, pp. 3243-3254, 2010.

[43] S. Tabibian, A. Akbari, and B. Nasersharif, "Speech enhancement using a wavelet thresholding method based on symmetric kullback-leibler divergence," Signal Processing, vol. 106, pp. 184-197, 2015.

[44] S. Tabibian, A. Akbari, and B. Nasersharif, "A new wavelet thresholding method for speech enhancement based on symmetric kullback-leibler divergence," in Computer conference, 2009. CSICC 2009. 14th international CSI, pp. 495-500, IEEE, 2009.

[45] H. Fan, F. Xie, Y. Li, Z. Jiang, and J. Liu, "Automatic segmentation of dermoscopy images using saliency combined with otsu threshold," Computers in Biology and Medicine, vol. 85, pp. 75-85, 2017.

[46] R. P. Braun, H. S. Rabinovitz, M. Oliviero, A. W. Kopf, and J.-H. Saurat, "Dermoscopy of pigmented skin lesions," Journal of the American Academy of Dermatology, vol. 52, no. 1, pp. 109-121, 2005.

[47] T. Ojala, M. Pietikäinen, and T. Mäenpää, "Multiresolution gray-scale and rotation invariant texture classification with local binary patterns," Pattern Analysis and Machine Intelligence, IEEE Transactions on, vol. 24, no. 7, pp. 971-987, 2002.

[48] L. Sørensen, S. B. Shaker, and M. De Bruijne, "Quantitative analysis of pulmonary emphysema using local binary patterns," Medical Imaging, IEEE Transactions on, vol. 29, no. 2, pp. 559-569, 2010.

[49] S. Naeem, F. Riaz, A. Hassan, and R. Nisar, "Description of visual content in dermoscopy images using joint histogram of multiresolution local binary patterns and local contrast," in International Conference on Intelligent Data Engineering and Automated Learning, pp. 433-440, Springer, 2015.

[50] T. Mendonca, P. M. Ferreira, J. S. Marques, A. R. Marcal, and J. Rozeira, "Ph 2-a dermoscopic image database for research and benchmarking," in Engineering in Medicine and Biology Society (EMBC), 2013 35th Annual International Conference of the IEEE, pp. 5437-5440, IEEE, 2013.

[51] ISIC Archive, The International Skin Imaging Collaboration: Melanoma Project. Available: https://isic-archive.com/. Online; Last accessed 04 January 2018

[52] C. Li, R. Huang, Z. Ding, J. C. Gatenby, D. N. Metaxas, and J. C. Gore, "A level set method for image segmentation in the presence of intensity inhomogeneities with application to mri," IEEE Transactions on Image Processing, vol. 20, no. 7, pp. 2007-2016, 2011.

[53] F. Riaz, A. Hassan, and J. Zeb, "Distance regularized curve evolution: A formulation using creaseness features for dermoscopic image segmentation," in Signal Processing (ICSP), 2014 12th IEEE International Conference on, pp. 1061-1065, IEEE, 2014.

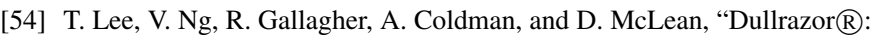
A software approach to hair removal from images," Computers in biology and medicine, vol. 27, no. 6, pp. 533-543, 1997.

[55] E. Ahn, L. Bi, Y. H. Jung, J. Kim, C. Li, M. Fulham, and D. D. Feng, "Automated saliency-based lesion segmentation in dermoscopic images," Engineering in Medicine and Biology Society (EMBC), 2015 37th Annual International Conference of the IEEE, pp. 3009-3012, 2015.

[56] R. Kasmi, K. Mokrani, R. Rader, J. Cole, and W. Stoecker, "Biologically inspired skin lesion segmentation using a geodesic active contour technique," Skin Research and Technology, vol. 22, no. 2, pp. 208-222, 2016.

[57] C. J. V. Rijsbergen, Information Retrieval. Butterworth-Heinemann, Newton, MA, USA, 1979.

[58] N. K. Mishra and M. E. Celebi, "An overview of melanoma detection in dermoscopy images using image processing and machine learning," arXiv preprint arXiv:1601.07843, 2016.

[59] E. Ahn, J. Kim, L. Bi, A. Kumar, C. Li, M. Fulham, and D. Feng, "Saliency-based lesion segmentation via background detection in dermoscopic images," IEEE journal of biomedical and health informatics, 2017.

[60] O. Michailovich, Y. Rathi, and A. Tannenbaum, "Image segmentation using active contours driven by the bhattacharyya gradient flow," IEEE Transactions on Image Processing, vol. 16, no. 11, pp. 2787-2801, 2007. 